
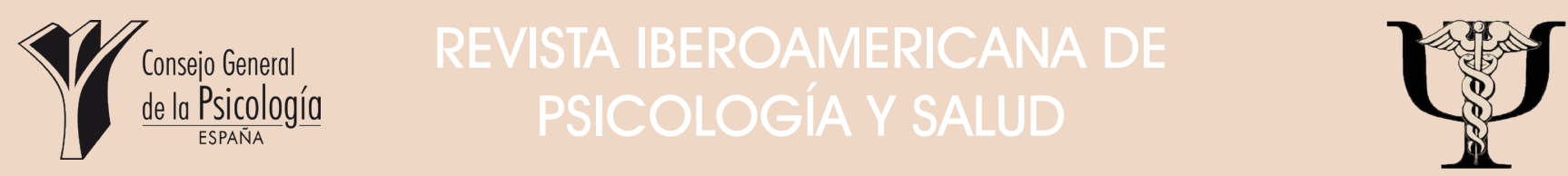

Revista Oficial de la Federación Iberoamericana de Asociaciones de Psicología (FIAP) [Official Journal of the Latin-American Federation of Psychological Associations]

\title{
Empatía y delincuencia juvenil: Un meta-análisis sobre la relación
}

\author{
Laura Férriz*, Jorge Sobral y José Antonio Gómez-Fraguela \\ Universidad de Santiago de Compostela.
}

- Recibido: 24 - 03 - 2017 - Aceptado: 13 - 07 - 2017 - Avance online: 18 - 12 - 2017

RESUMEN. El objetivo principal de esta investigación es estudiar la relación ente empatía y delincuencia oficialmente registrada durante la adolescencia. Se llevó a cabo mediante una revisión sistemática de todos aquellos estudios obtenidos a través de diversas estrategias de búsqueda. Además, se ha incorporado una distinción clave en el campo, a menudo olvidada, diferenciando entre empatía afectiva y cognitiva. Entre los criterios de inclusión se encuentra uno que resulta clave: la presencia de delincuencia registrada como tal por la ley, excluyendo todos aquellos comportamientos vandálicos o de naturaleza antisocial. Como resultado, se obtuvo una muestra de $k=39$ estudios primarios para la empatía cognitiva y $k=52$ estudios para la afectiva, abarcando más de 30 años de investigación. Los resultados revelan una asociación moderada entre delincuencia y la dimensión cognitiva $(r=-.220)$ y algo más leve con la dimensión afectiva ( $r=$-. 140). Se realizó un análisis de moderadores observando que determinadas características (tanto sociodemográficas como metodológicas) modulan la intensidad de la asociación entre empatía y delincuencia. Se discuten las implicaciones para futuras investigaciones así como su importancia para el desarrollo sociopsicológico de esta población en específico.

PALABRAS CLAVE: Empatía, Delincuencia, Adolescencia, Meta-análisis, Revisión.

\section{Empathy and juvenile delinquency: A meta-analytic review.}

ABSTRACT. The primay aim of this review was to study existing knowledge on the relationship between empathy and juvenile official delinquency. We conducted a meta-analytic review taking into account the differences between cognitive empathy and affective empathy. Only those studies based on official delinquency records in the applicable country were included. Therefore, all studies focusing on antisocial behaviors, traits such as aggressiveness and/or behavioral problems were excluded. As a result, we retrieved a total of 39 studies about the relationship between cognitive empathy and delinquency, and 52 studies about the affective empathy. The results found a moderated effect size $(r=-.220)$ in the case of cognitive empathy and quite lower effect size for the affective empathy $(r=-.140)$. We also analyzed a set of moderating variables such as methodologic and sociodemographic variables. These variables have a potential moderating effect. Its importance on future researches as well as on the sociopsychological development of this population are disscussed.

KEYWORDS: Empathy, Delinquency, Adolescence, Meta-analytic, Review.

La empatía se configura como una capacidad clave en el desarrollo de pensamientos y conductas adaptadas a los contextos sociales (García-López, del Campo, Gutiérrez, GonzálezVillora y Valenzuela, 2012). Por ello, la empatía

\footnotetext{
*Correspondencia: Laura Férriz Romeral

Universidad de Santiago de Compostela.

Código Postal: 15782, Calle Xosé María Suárez Núñez, s/n.

Campus Vida

E-mail: laura.ferriz@rai.usc.es
}

(C) 2018 Sociedad Universitaria de Investigación en Psicología y Salud. Publicado por Consejo General de Colegios Oficiales de Psicologos, España. Este es un artículo Open Access bajo la CC BY-NC-ND licencia (http://Creativeconm

Citar como/Cite as: Férriz, L., Sobral, J., \& Gómez. A. J. (2018). Empatía y delincuencia juvenil: Un meta-análisis sobre la relación. Revista Iberoamericana de Psicología y Salud, 911), 1-16. https://doi.org/10.23923/i.rips.2018.01.011 resulta crucial en el desarrollo, tanto a nivel cognitivo como comportamental. Asimismo, aunque sea susceptible de ser abordada monográficamente, no es una variable individual con una evolución ontogenética aislada de otras, sino que se integra en una compleja red de interacciones.

De este modo, se ha analizado y corroborado la relación existente entre empatía y conductas antisociales diversas en numerosos estudios (Calvo, González y Martorell, 2001; Li 
et al., 2015; Navas, Muñoz y Graña, 2005). En sentido positivo, desde hace varias décadas se ha mostrado el importante papel de la empatía, como factor motivacional de las conductas prosociales, ya desde edades muy tempranas (Eisenberg, Fabes, Guthrie y Reiser, 2000; Goutaudier, Lopez, Belarbi, Ducroux y Chabrol, 2015).

En relación a la delincuencia, la mayoría de estudios se han percatado de la presencia de niveles bajos de empatía en menores infractores (ej.: Burke, 2001; Chandler y Moran, 1990; González-Gadea et al., 2014), aunque estas diferencias no han sido obtenidas en todos los estudios realizados (ej.: Lee y Prentice, 1988, Kaplan y Arbuthnot, 1985).

Todo ello motivó la realización del meta-análisis llevado a cabo por Jolliffe y Farrington (2004). En él se incluye estudios muy heterogéneos entre sí, desde algunos comparando muestras de delincuentes violentos con delincuentes no violentos hasta estudios primarios cuya población objetivo son delincuentes con problemas mentales. Además, no se centra en poblaciones adolescentes, las cuales tienen unas singularidades bien conocidas en relación con ciertas conductas problemáticas (Martorell, González, Ordóñez y Gómez, 2011). Asimismo, no tiene en cuenta el posible efecto moderador de variables tanto metodológicas como demográficas (como la edad), siendo esta una diferencia clave entre el presente estudio y dicho meta-análisis.

De este modo, entre estas posibles variables moderadoras, resulta necesario tener en cuenta la edad, pues parece haber consenso en que el nivel de empatía aumenta durante la adolescencia, siendo especialmente relevante el incremento de la empatía cognitiva (Allemand, Steiger y Fend, 2015; Schwenck et al., 2014), es necesario determinar si la importancia del papel que juega la empatía como factor motivacional de determinados comportamientos también incrementa con la edad. Para el caso del papel que juega el sexo en el nivel de empatía, no parece haber un especial acuerdo. De este modo, hay autores que observan diferencias significativas entre ambos sexos teniendo las chicas niveles más altos de empatía (Stuijfzand et al., 2016); no obstante, otros autores no han encontrado evidencia sobre estas diferencias (Euler, Steinlin y Stadler, 2017). También es necesario diferenciar entre los estudios en función del instrumento de medida de la empatía empleado, pues cada uno de ellos se acerca al constructo de empatía desde diferentes perspectivas (cognitiva 0 afectiva). Asimismo, tal como afirman Colby y Kohlberg (1987), el medio cerrado sería capaz de generar una atmósfera autocentrada reduciendo el nivel de razonamiento moral en el menor. Extendiendo esta reflexión al nivel de empatía de menores institucionalizados, se torna oportuno tener en cuenta el método de medida de la delincuencia (comparación de menores institucionalizados con grupo control o empleo de otras medidas).

Por último, numerosos autores se han percatado del hecho de que niveles más bajos de empatía se encuentra relacionado de un modo más intenso con determinados delitos, especialmente con delitos contra personas (Van der Graaff et al., 2014), o el hecho de que la empatía tiene un papel relevante en determinados delitos (Brewer y Kerslake, 2015).

Por tanto, el objetivo principal del presente estudio es obtener un valor actualizado que resuma casi cuatro décadas de investigación de la relación entre los comportamientos delictivos en jóvenes en la etapa de la adolescencia y el grado de empatía que presentan los mismos. Además, se propone examinar si el tipo de empatía (cognitiva y/o afectiva) provoca variaciones en el grado de intensidad de esa relación, así como conocer si los instrumentos de medida empleados y variables como el sexo, la edad y el tipo de delito podrían estar modulando dicha relación, algo no tenido en cuenta en el meta-análisis de Jolliffe y Farrington (2004).

\section{MÉTODO}

\section{- DISEÑO Y PROCEDIMIENTO}

Se han llevado a cabo numerosas estrategias en la búsqueda y localización de posibles estudios primarios candidatos para formar parte de la revisión.

En primer lugar, se realizó una búsqueda en las bases de datos PsyclNFO, Web of Science, ERIC, ProQuest Dissertations \& Theses Global, Redalyc, Dialnet y CSIC, para lo cual se combinaron los siguientes descriptores: empathy, delinquen*, offen* and crim* sin restricciones de fecha o idioma. Además, se buscó en estas bases de datos el nombre de los 
principales investigadores en el campo. Tras esto, se emplearon los mismos descriptores en el metabuscador Google Schoolar. La tercera estrategia consistió en revisar las referencias bibliográficas de todos los estudios obtenidos con los procedimientos anteriores, así como las referencias de las revisiones cuantitativas y narrativas localizadas sobre la temática. Además, se estableció contacto con investigadores que trabajan en la misma línea con el fin de obtener estudios que no hayan sido publicados, previniendo así sesgos en nuestros resultados.

\section{- CRITERIOS DE INCLUSIÓN}

A la hora de determinar la inclusión de un estudio primario como parte del metaanálisis, éste debía cumplir los siguientes criterios: la muestra empleada debía tener una edad comprendida entre los 11 y los 20 años, y en segundo lugar, debía incluir un tamaño de efecto o cualquier otra medida susceptible de ser convertida a un tamaño de efecto que relacionarse las variables empatía y delito.

Por su parte, se hizo hincapié en la inclusión de la conocida como grey literature, así como la no distinción por idioma o periodo de tiempo en el procedimiento de búsqueda.

\section{- CRITERIOS DE EXCLUSIÓN}

En cuanto a la delincuencia, fueron excluidos los estudios centrados únicamente en comportamientos antisociales que no constituyen un delito, o aquellos estudios con poblaciones con cualquier tipo de problema mental. Además, se excluyeron los estudios centrados en delitos relacionados con drogas, por su especificidad.

\section{- RECOLECCIÓN DE DATOS}

Una vez obtenidos los estudios primarios, se llevó a cabo un protocolo de codificación tanto de las características substantivas como metodológicas de cada estudio. Dicho protocolo de codificación fue llevado a cabo mediante una hoja de codificación rellenada por duplicado por dos investigadores independientes con los siguientes datos: año del estudio, tamaño de la muestra, edad de la muestra, sexo, método de medida de la variable "empatía", método de medida de la variable "delincuencia", tipo de empatía (general, cognitiva o afectiva), y tipo de delito. Los desacuerdos fueron discutidos entre los jueces llegando a una conclusión; en aquellos casos en los que no fue posible, un tercer juez fue quien decidió la opción más acertada.

\section{RESULTADOS}

\section{- REFERENCIAS SELECCIONADAS}

Tras el procedimiento de búsqueda y codificación de los estudios (el acuerdo entre codificadores medida mediante la Kappa de Cohen resultó ser .89), se obtuvieron un total de trescientos trece artículos, que tras eliminar los ciento veintinueve artículos duplicados, se redujeron a ciento ochenta y cuatro. De estos, ciento dieciocho estudios fueron excluidos por no cumplir con los criterios exigidos para formar parte de la revisión. Finalmente, el número de estudios independientes encontrados para la relación de delincuencia y empatía desde un punto de vista multidimensional fue de setenta y cuatro. No obstante, este número se vio reducido a sesenta y nueve tras la eliminación de los estudios cuyos resultados eran outliers que sesgaban los resultados de la revisión. Sobre la relación entre delincuencia y empatía cognitiva, se reunieron un total de treinta y nueve estudios independientes, y para el caso de la empatía afectiva, cincuenta y dos estudios (tras la eliminación de los outliers). La ilustración 1 muestra el diagrama de flujo del proceso de revisión sistemática.

\section{- ANÁLISIS DE CONTENIDO DE LOS ESTUDIOS INCLUIDOS}

La muestra total fue de diecinueve mil ciento trece menores, con una edad media de 16 años. En concreto, los estudios referentes a empatía cognitiva reunieron un total de diez mil novecientos sesenta y cinco menores mientras que en el caso de la empatía afectiva se obtuvo una muestra de nueve mil seiscientos treinta menores. Los estudios obtenidos referentes a ambos tipos de empatía junto a sus principales características aparecen en el tabla 1. 


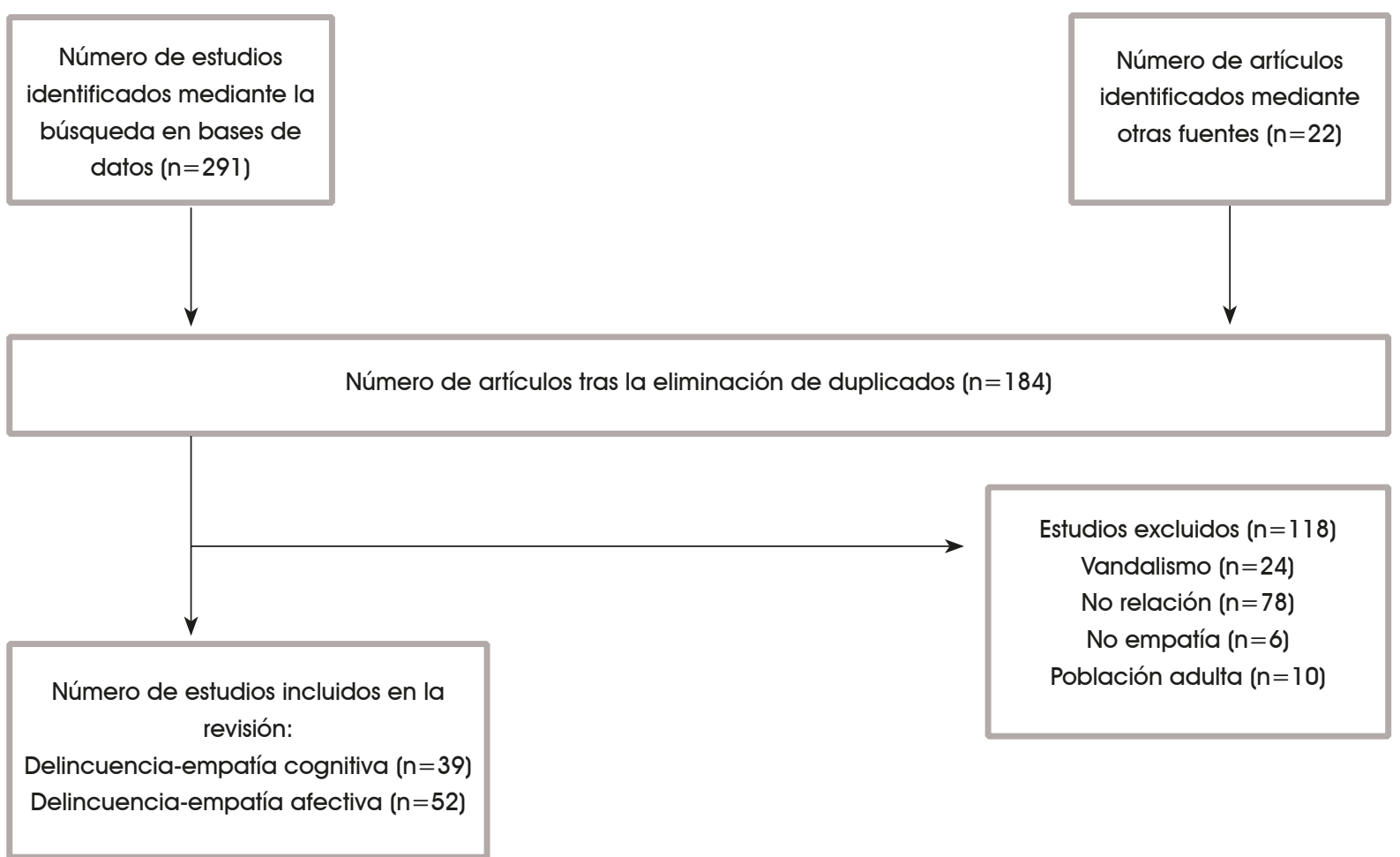

Figura 1. Diagrama de flujo del proceso de revisión sistemática. De los estudios excluidos, 24 de ellos lo fueron debido a su referencia a actos vandálicos. Además, 78 estudios fueron desestimados por no contener un dato cuantitativo sobre la relación entre delincuencia y empatía. Por último, de los restantes estudios, 10 fueron excluidos por emplear una muestra de adultos y 6 lo fueron por tratar conceptos distintos a la empatía.

Tabla 7

Estudios incluidos en la revisión y características

\begin{tabular}{|c|c|c|c|c|c|}
\hline AUTOR & AÑO & $\mathrm{N}$ & SEXO & EMP. COGNITIVA & EMP. AFECTIVA \\
\hline Ashraf, Khalid y Ahmed & 2014 & 125 & Hombre & & -.28 \\
\hline $\begin{array}{l}\text { Broidy, Cauffman, } \\
\text { Espelage, Mazerolle y } \\
\text { Piquero }\end{array}$ & 2003 & 348 & Mujer & & -.25 \\
\hline Broidy et al. & 2003 & 309 & Hombre & & -.2 \\
\hline Burke & 2001 & 46 & Hombre & -.39 & -.31 \\
\hline Bush & 1997 & 109 & Hombre & .06 & .14 \\
\hline Bush & 1997 & 66 & Mujer & .08 & .13 \\
\hline Bush, Mullis y Mullis & 2000 & 175 & Mixto & .04 & .06 \\
\hline Caruso & 2003 & 10 & Hombre & .21 & \\
\hline Chandler y Moran & 1990 & 80 & Hombre & -.36 & \\
\hline Costa & 2003 & 647 & Hombre & -.29 & -.26 \\
\hline
\end{tabular}


Tabla 7 (Continuación)

Estudios incluidos en la revisión y características

\begin{tabular}{|c|c|c|c|c|c|}
\hline AUTOR & AÑO & $\mathrm{N}$ & SEXO & EMP. COGNITIVA & EMP. AFECTIVA \\
\hline D'Antonio & 1997 & 105 & Mixło & .11 & -.2 \\
\hline $\begin{array}{l}\text { De Kemp, Overbeek, } \\
\text { Wied, Engels y Scholte }\end{array}$ & 2007 & 403 & Hombre & & -.19 \\
\hline De Kemp et al & 2007 & 420 & Mujer & & -.07 \\
\hline D'Orazio & 2002 & 60 & Hombre & .19 & .04 \\
\hline Ellis & 1980 & 201 & Hombre & -.35 & \\
\hline Ellis & 1980 & 145 & Hombre & -.03 & \\
\hline Forbes & 2003 & 199 & Mixło & & -.02 \\
\hline González-Gadea & 2014 & 46 & Hombre & & -.13 \\
\hline Goodman & 1998 & 102 & Hombre & & -.25 \\
\hline Granado & 2014 & 50 & Hombre & & -.28 \\
\hline Gray & 1997 & 101 & Hombre & & -.07 \\
\hline Hackenberg & 2002 & 259 & Hombre & -.26 & -.16 \\
\hline $\begin{array}{l}\text { Hepper, Hart, Meek, } \\
\text { Cisek y Sedikides }\end{array}$ & 2014 & 146 & Hombre & -.08 & -.24 \\
\hline Hudak & 1978 & 96 & Mixto & -.12 & \\
\hline Hudak & 1980 & 64 & Mixło & -.04 & \\
\hline $\begin{array}{l}\text { Hunter, Figueredo, } \\
\text { Becker y Malamuth }\end{array}$ & 2007 & 184 & Hombre & & -.07 \\
\hline Jolliffe y Farrington & 2007 & 376 & Hombre & -.08 & -.12 \\
\hline Jolliffe y Farrington & 2007 & 344 & Mujer & .02 & 0 \\
\hline Jolliffe y Farrington & 2007 & 376 & Hombre & & -.08 \\
\hline Jolliffe y Farrington & 2007 & 344 & Mujer & & -.2 \\
\hline Kaplan y Arbuthnot & 1985 & 40 & Mixto & & 0 \\
\hline Kelley & 1992 & 30 & Mixto & & -.35 \\
\hline $\begin{array}{l}\text { Kendall, Deardorff y } \\
\text { Finch }\end{array}$ & 1977 & 48 & Hombre & -.29 & \\
\hline $\begin{array}{l}\text { Larden, Melin, Holst y } \\
\text { Langstrom }\end{array}$ & 2006 & 58 & Hombre & & -.09 \\
\hline Larden et al & 2006 & 58 & Mujer & & 0 \\
\hline Lauterbach y Hosser & 2007 & 839 & Hombre & -.08 & -.04 \\
\hline Lee y Prentice & 1988 & 54 & Hombre & & .05 \\
\hline Lindsey & 1997 & 54 & Hombre & -.23 & -.26 \\
\hline Lindsey, Carlozzi y Eells & 2001 & 81 & Hombre & -.18 & .01 \\
\hline Lindsey et al. & 2001 & 54 & Hombre & -.02 & .09 \\
\hline Lo & 2007 & 102 & Hombre & -.33 & -.1 \\
\hline MacBride & 2005 & 394 & Mixło & -.22 & -.12 \\
\hline Mak & 1991 & 206 & Mixło & & -.11 \\
\hline Malgorzata & 1997 & 55 & Hombre & & -13 \\
\hline McCrady & 2005 & 210 & Hombre & -.05 & .02 \\
\hline
\end{tabular}




\begin{tabular}{|c|c|c|c|c|c|}
\hline AUTOR & AÑO & $\mathrm{N}$ & SEXO & EMP. COGNITIVA & EMP. AFECTIVA \\
\hline Milojevic y Dimitrijevic & 2014 & 90 & Hombre & -.14 & .18 \\
\hline $\begin{array}{l}\text { Moriarty, Stough, } \\
\text { Tidmarsh, Eger y } \\
\text { Dennison }\end{array}$ & 2001 & 64 & Hombre & -.18 & -.08 \\
\hline Park & 2003 & 48 & Mujer & & .04 \\
\hline Reyes & 1990 & 32 & Hombre & -.19 & \\
\hline Reyes & 1990 & 32 & Hombre & -.25 & \\
\hline Reynolds & 1999 & 215 & Hombre & -.17 & -.06 \\
\hline Riley & 1986 & 3953 & Mixto & -.23 & \\
\hline $\begin{array}{l}\text { Robinson, Roberts, } \\
\text { Strayer y Koopman }\end{array}$ & 2007 & 124 & Hombre & -.2 & -.06 \\
\hline $\begin{array}{l}\text { See, Ang, Loh, Fu y } \\
\text { Karre }\end{array}$ & 2011 & 241 & Hombre & -.11 & -.24 \\
\hline Stams et al & 2008 & 195 & Mixto & & .04 \\
\hline Sturgeon & 2003 & 303 & Hombre & -.06 & -.07 \\
\hline Trivits & 2005 & 117 & Mujer & .04 & .1 \\
\hline $\begin{array}{l}\text { Van der Graaff, Branje, } \\
\text { De Wled y Meeus }\end{array}$ & 2012 & 323 & Mixto & & -.25 \\
\hline Van Outsen et al. & 2006 & 60 & Hombre & -.33 & -.25 \\
\hline Way & 1999 & 96 & Hombre & -.24 & .01 \\
\hline Way & 1999 & 89 & Hombre & .2 & .02 \\
\hline Way & 1999 & 51 & Hombre & 1 & -.01 \\
\hline
\end{tabular}

Del cálculo del tamaño de efecto para la relación entre empatía (entendida desde un enfoque multidimensional) y delincuencia una vez corregido de los errores artifactuales (ponderado por el tamaño de la muestra y corrección por atenuación tanto del criterio como del predictor), empleando el modelo de efectos fijos se obtuvo una correlación negativa de $r=-.212, p<.001$; 95\% IC [-.227, -. 198]. Al llevar a cabo el test de homogeneidad, se observa que $Q(68)=284.43$, $p<.0001$. Este resultado indica la posible presencia de una serie de variables moderadoras.

Para el caso de la empatía cognitiva y delincuencia, el tamaño de efecto corregido fue $r=-.220, p<.001 ; 95 \%$ IC [-.240,-.200] mientras que en el caso de la empatía afectiva, el tamaño de efecto fue algo menor $r=-.140, p<.001 ; 95 \%$ IC [-. 160, -.120]. El análisis de homogeneidad tanto para la empatía cognitiva como afectiva muestra una alta variabilidad en los resultados, $Q(39)=136.53$, $p<.001$ y $Q(52)=120.16, p<.001$, respectivamente.
Debido a ello, procedimos a analizar la presencia de variables moderadoras.

El ANOVA realizado mediante el modelo de efectos fijos mostró la presencia de 3 variables moderadoras significativas para el caso de la empatía cognitiva. Los resultados obtenidos se presentan en la tabla 2. De este modo, la edad resultó ser una variable moderadora significativa $(Q(34)=28.30, p<.001)$. El tamaño de efecto de la empatía cognitiva para el caso de menores de entre 15 y 16 años se mostró mayor $(r=-.193, p<.001 ; 95 \%$ IC $[-.215,-.171])$ que para aquellos menores con más de 16 años $(r=-.067, p<.01 ; 95 \%$ IC [-. 113, -.020]). En el caso del sexo, el modelo de efectos fijos mostró dicha variable como moderadora del tamaño del efecto $(Q(38)=30.47, p<.001)$. El tamaño de efecto para la empatía cognitiva para hombres ( $r=-.147, p<.001 ; 95 \%$ IC $[-.175,-.119])$ es mayor que el tamaño de efecto resultante para mujeres ( $r=-.032$, n.s.; 95\% IC [-.053, -. 117$]$ ). 
Tabla 2

ANOVA univariante entre tamaño de efecto para empatía cognitiva y delincuencia así como variables moderadoras

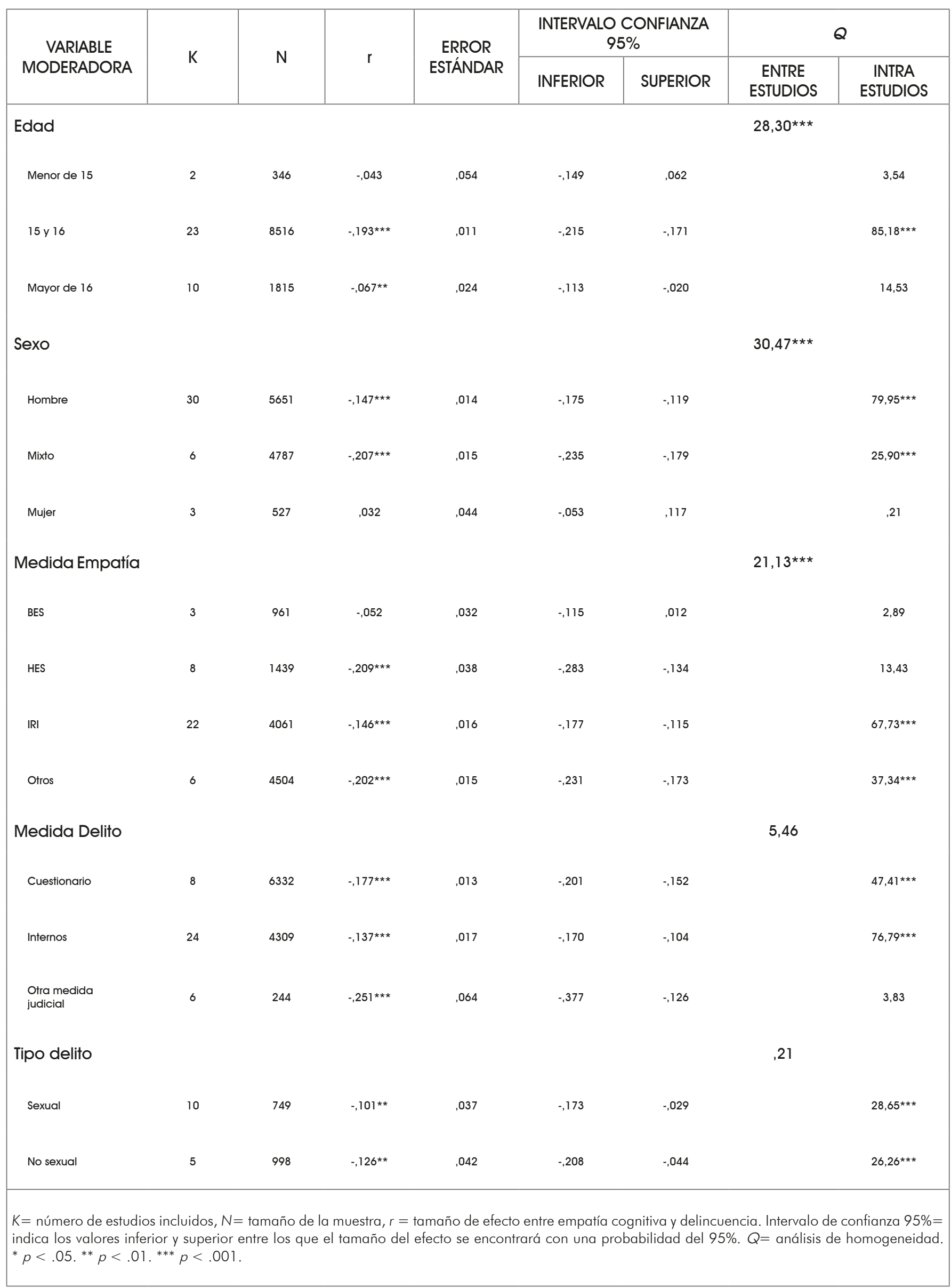


Tabla 3

ANOVA univariante entre tamaño de efecto para empatía afectiva y delincuencia así como variables moderadoras

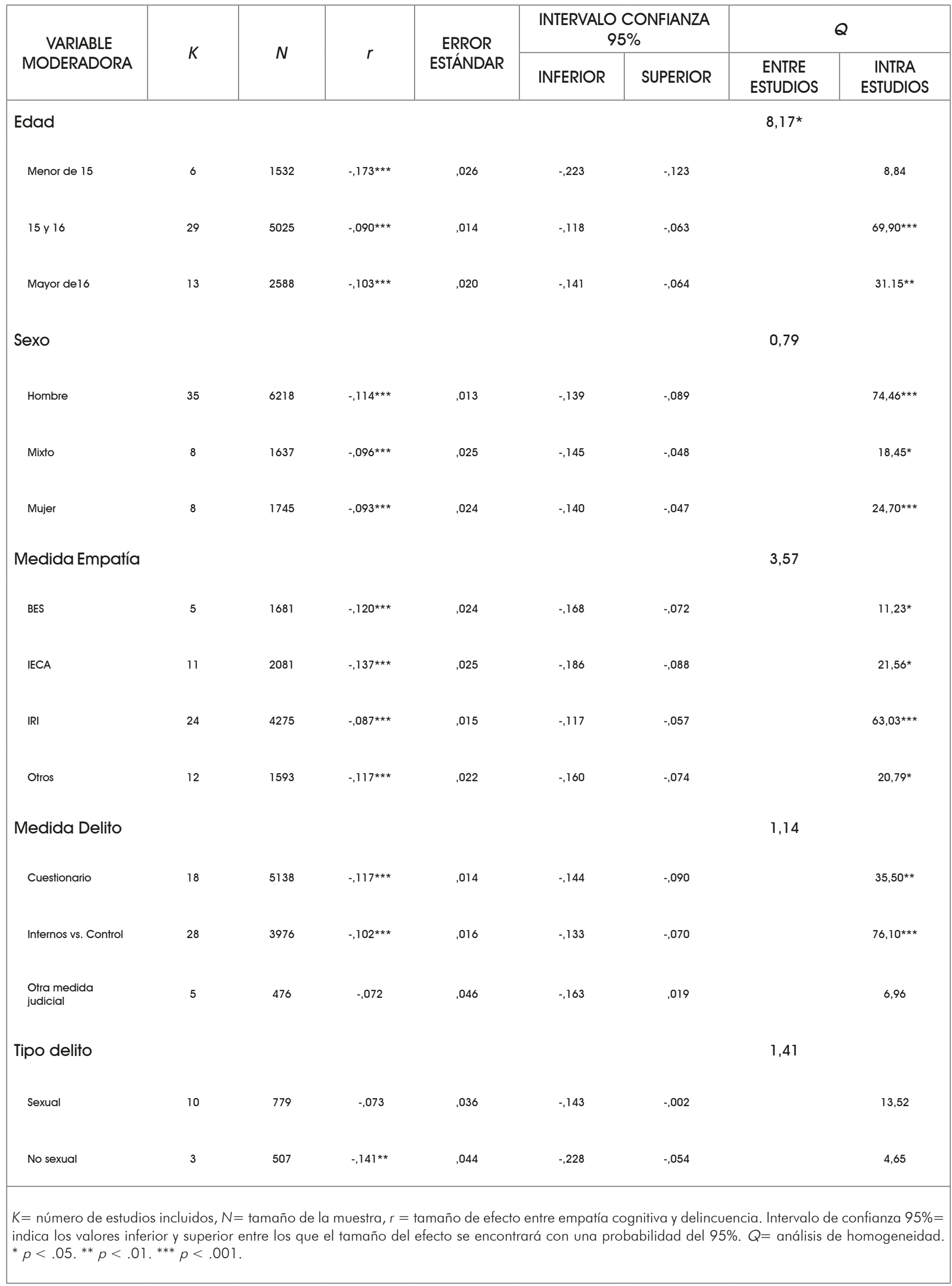


En cuanto a las características metodológicas, la medida empleada para evaluar la empatía cognitiva resultó ser un moderador de la relación $(Q(38)=21.13$, $p<.001)$. El empleo del cuestionario Hogan Empathy Scale (HES) de Hogan (1969) generó un tamaño de efecto más alto $(r=-.209$, $p<.001 ; 95 \%$ IC $[-.283,-.134])$ que el uso de las dimensiones toma de perspectiva y fantasía del Interpersonal Reactivity Index (IRI) de Davis (1980) $(r=-.146, p<.001$; 95\% IC [-.177, -. 115$]$ ), el Basic Empathy Scale (BES) de Jolliffe y Farrington (2006) ( $r=-.052$, n.s.; 95\% IC [-. 115 , -.012]) entre otros. En cuanto al procedimiento de registro del delito, no se mostró con un efecto moderador significativo, encontrando homogeneidad en los tamaños de efecto independientemente del uso de cuestionarios o el empleo de muestras de sujetos privados de libertad o en cumplimiento de medidas judiciales alternativas al internamiento. Lo mismo ocurre en el caso de la variable referente al tipo de delito, pues no se muestra como una variable moderadora significativa $(Q(14)=.21$; $p<.65)$.

En el caso de la empatía afectiva, ésta se mostró reacia a la influencia moderadora, pues tan sólo la edad fue detectada por el modelo de efectos fijos como variable moderadora $(Q(47)=8.18, p<.05)$, mostrando que los adolescentes de menos de 15 años muestran un tamaño de efecto mayor para la empatía afectiva $(r=-.173, p<.001 ; 95 \%$ IC $[-.223,-.123])$ que los chicos de más de 15 años ( $r=-.090, p<.001 ; 95 \%$ IC [-. 118, -.063]). El resto de variables no fueron significativamente moderadoras. Los resultados obtenidos mediante el modelo de efectos fijos se muestran en la tabla 3.

Por último, se mostró la ausencia del "file drawer problem" (Rosenthal, 1979) mediante el cálculo del filesafe $N$ cuyo resultado fue de $N=192$ estudios, lo que muestra que las conclusiones del presente meta-análisis no se ven afectadas por el sesgo de publicación.

\section{DISCUSIÓN Y CONCLUSIONES}

El objetivo principal de este estudio fue esclarecer la relación entre empatía y delincuencia en jóvenes, distinguiendo además entre sus dimensiones cognitiva y afectiva. Es necesario aclarar que de la relación entre ambas variables mostrada en este trabajo, no es posible inferir una determinación causal entre ellas.

En cuanto a los resultados, se obtiene una relación significativa y negativa entre la empatía y la delincuencia $(r=-.212, p<.001)$, de modo que los menores infractores presentan niveles de empatía más bajos que aquellos que no cometieron delitos.

Más específicamente, se encuentra que la empatía cognitiva correlaciona con la delincuencia $(r=-.220, p<.001)$ con una mayor intensidad que la empatía afectiva $(r=-.140, p<.001)$. Este resultado indicaría déficits más profundos de empatía cognitiva que de empatía afectiva, de modo que el principal problema con el que se enfrentarán estos menores es en llegar a comprender y entender los estados internos de los demás, y no tanto en su capacidad para experimentar ellos mismos estados emocionales similares en alto grado a los de otros (por ejemplo, personas en situación de sufrimiento). Este hecho provocaría que estos menores se encontrarían limitados a la hora de experimentar dichos estados emocionales por sus pensamientos y cogniciones.

En cuanto a los resultados asociados a la edad, en el caso de la empatía cognitiva, los adolescentes entre 15 y 16 años obtienen tamaños de efectos mayores $(r=-.193, p<$ $.001)$, que aquellos de más edad ( $r=-.067$, $p<.01)$. Para la empatía afectiva, los chicos menores de 15 años obtuvieron tamaños de efecto mayores $(r=-.173, p<.001)$ que chicos de mayor edad $(r=-.090, p<.001)$. Según estos resultados, tanto la empatía cognitiva como la empatía afectiva, mostrarían una relación moderada con comportamientos delictivos a edades muy tempranas, perdiendo intensidad con la edad. Ello mostraría la especial importancia de la empatía, pues es en los primeros años de la adolescencia cuando los menores pueden llegar a cometer los primeros actos ilegales y la empatía podría servir como freno de las mismas.

Por su parte, no aparecen diferencias significativas en la relación entre empatía afectiva y delincuencia según el sexo, pero sí para el caso de la relación con la empatía cognitiva, en la que los chicos mostrarían tamaños de efecto mayores que las chicas. En este sentido, está constatado empíricamente que los hombres 
suelen presentar sistemáticamente niveles más bajos de empatía cognitiva que las mujeres, independientemente de la edad (Hoffman, 1977; Eisenberg y Lennon, 1983).

En cuanto a los instrumentos de evaluación, el empleo del cuestionario HES para medir empatía cognitiva muestra tamaños de efecto sensiblemente mayores $(r=-.209, p<.001)$ que el uso de otros como el IRI $(r=-.146$, $p<.001)$. De acuerdo con Jolliffe y Farrington (2004), ello se debería a las características del HES, pues este posee un diseño de respuesta de verdadero/falso, lo cual reduce la variabilidad en las respuestas. Además, Vachon, Lynam y Johnson (2014) consideran que el HES contiene ítems referentes a rasgos externalizantes (ej.: "a veces me divierto hiriendo a las personas que quiero" o "a veces me divierte ir en contra de las reglas") lo cual incrementa la capacidad de este instrumento para predecir comportamientos problemáticos.

Respecto al procedimiento de registro de la delincuencia, no se observaron variaciones en los tamaños de efecto según el método empleado. Parece ser indiferente el empleo de cuestionarios para evaluar la delincuencia o el uso de muestras de menores institucionalizados.

Además del análisis aquí realizado, sería prioritario profundizar en las relaciones que la investigación entre la empatía y la delincuencia debería establecer con ámbitos propios de la psicología del desarrollo moral, del razonamiento moral y hasta de la filosofía moral. De modo que en ese campo debe haber buenas respuestas acerca de qué mecanismos últimos conforman la decisión de delinquir o no hacerlo, al menos en la medida en qué esa decisión pueda ser fruto de algún nivel de libre albedrío (Sobral y Gómez-Fraguela, 2015).

A nivel práctico, es necesario emplear todo el conocimiento reunido sobre la empatía para la prevención y evitación de comportamientos negativos en niños y adolescentes. Una de las problemáticas con un mayor aumento de su incidencia entre los jóvenes en los últimos años es el bullying. La empatía puede jugar un especial papel preventivo, e incluso predictivo de este gran problema que afecta a tantos menores en todo el mundo (Del Rey et al., 2016).

A modo de conclusión, teniendo en cuenta la notable influencia de la empatía sobre el comportamiento humano en general, es evidente, pues, la necesidad de tenerla muy presente en la educación y desarrollo de niños y adolescentes, ya sean menores inmersos en procesos judiciales o no, facilitando que la empatía juegue su rol como instrumento de desarrollo armónico y prosocial de su personalidad.

\section{- Conflicto de intereses}

Los autores declaran no tener ningún conflicto de intereses.

\section{REFERENCIAS}

Las referencias marcadas con un asterisco $\left({ }^{*}\right)$ son los estudios incluidos en el metaanálisis.

Allemand, M., Steiger, A. E., y Fend, H. A. (2015). Empathy development in adolescence predicts social competencies in adulthood. Journal of Personality, 83(2), 229-241. http://dx.doi. org/10.1111/jopy. 12098

* Arundell, R.M. (1992). The relationships of empathy, social support, trauma symptoms and family violence in the victim to victimizer process in adolescent males. Tesis doctoral. University of Cincinnati, Cincinnati. Recuperado de https://search.proquest.com/ docview/618197983? accountid =17253

* Ashraf, S., Khalid, S., y Ahmed, F. (Junio, 2014). A study of emotional empathy and delinquency. En J. Shopovski (Presidencia), advancing knowledge through interdisciplinary research. Simposio llevado a cabo en el I International Conference on Social Sciences and Humanities, Gaborone, Botswana.

* Bandura, A., Caprara, G.V., Barbaranelli, C., Gerbino, M., y Pastorelli, C. (2003). Role of affective self-regulatory efficacy in diverse spheres of psychosocial functioning. Child Development, 74(3), 769-782. http://dx.doi. org/10.1111/1467-8624.00567

* Beail, N., y Ralfs, S. (2010). Victim specific empathy in offenders. Journal of Applied Research in Intellectual Disabilities, 23(5), 427-427. http://dx.doi.org/10.1111/ j.1468-3148.2010.00582.x

* Bechtoldt, H. (2006). The role of empathy for delinquent and non-delinquent adolescents 
exposed to violence: Is it protective?. Tesis doctoral. University of Toledo, Ohio, EEUU. Recuperado de https://search.proquest.com/ docview/621574545? accountid $=17253$

Brewer, G., y Kerslake, J. (2015). Cyberbullying, self-esteem, empathy and loneliness. Computers in Human Behavior, 48, 255-260. http://dx.doi.org/10.1016/i. chb.2015.01.073

* Broidy, L., Cauffman, E., Espelage, D.L., Mazerolle. P., y Piquero, A. (2003). Sex differences in empathy and its relation to juvenile offending. Violence and victims, 18(5), 503-516. http://dx.doi.org/10.1891/ vivi.2003.18.5.503

* Burke, D.M. (2001). Empathy in sexually offending and non-offending adolescent males. Journal of Interpersonal Violence, 16(3), 222-233. http://dx.doi. org/10.1177/088626001016003003

*Bush, C.A. (1997). Genderdifferencesinempathy for delinquent and nondelinquent youths. Tesis doctoral. Florida State University. Florida. Recuperado de https://search.proquest.com/ docview/304394823? accountid = 17253

* Bush, C.A., Mullis, R. L., y Mullis, A.K. (2000). Differences in empathy between offender and nonoffender youth. Journal of Youth and Adolescence, 29(4), 467-478. Recuperado de https://search.proquest.com/ docview/204521088? accountid = 17253

Calvo, A. J., González, R., y Martorell, M. C. (2001). Variables relacionadas con la conducta prosocial en la infancia y adolescencia: Personalidad, autoconcepto y género. Journal for the Study of Education and Development, 24(1), 95-111. http://dx.doi. org/10.1174/021037001316899947

* Carrasco, M., Baker, E.D., Tremblay, R.E., y Vitaro, F. (2006). Eysenck's personality dimensions as predictors of male adolescent trajectories of physical aggression, theft and vandalism. Personality and Individual Differences, 47 (7) 1309-1320. http://dx.doi. org/10.1016/i.paid.2006.05.005

* Chandler, M., y Moran, T. (1990). Psychopathy and moral development: A comparative study of delinquent and nondelinquent youth. Development and Psychopathology, 2(3), 227-246. http://dx.doi.org/10.1017/ S0954579400000742

Colby, A., y Kohlberg, L. (1987). The measurement of moral judgment, vol. 1: Theoretical foundations and research validation; vol. 2: Standard issue scoring manual. New York, EEUU: Cambridge University Press. Recuperado de https://search.proquest.com/ docview/617359193? accountid $=17253$

* Costa, M. (2003). Young offenders and social skills training. Tesis doctoral. Universidad de Toronto, Toronto, Canadá. Recuperado de https://search.proquest.com/ docview/305251347? accountid = 17253

* D'Antonio, A. M. (1997). The effect of empathy on aggression and antisocial behavior among low-impulsive and high-impulsive children and adolescents. Tesis doctoral. The George Washington University, Washington. Recuperado de https://search.proquest.com/ docview/619254366? accountid = 17253

* D'Orazio, D.M. (2004). Acomparative analysis of empathyin sexuallyoffending and nonoffending juvenile and adult males. Tesis doctoral. Alliant International University, California. Recuperado de https://search.proquest.com/ docview/620625795? accountid = 17253

Davis, M.H. (1979). Individual differences in empathy: A multidimensional approach. Tesis doctoral. Universidad de Texas, Austin. Recuperado de https://search.proquest.com/ docview/302984573? accountid $=17253$

* Deardorff, P. A., Finch, A. J., J., Kendall, P. C., Lira, F., \& Indrisano, V. (1975). Empathy and socialization in repeat offenders, first offenders, and normals. Journal of Counseling Psychology, 22(5), 453-455. http://dx.doi. org/10.1037/h0077127.

* Delhaye, M., Kempenaers, C., Stroobants, R., Goossens, L., y Linkowski, P. (2013). Attachment and socio-emotional skills: A comparison of depressed impatients, institutionalized delinquents and control adolescents. Clinical Psychology and Psychotherapy, 20(5), 424-433. Recuperado de https://search.proquest.com/ docview/1536024720? accountid $=17253$ 
Del Rey, R., Lazuras, L., Casas, J. A., Barkoukis, V., Ortega-Ruiz, R., y Tsorbatzoudis, $H$. (2016). Does empathy predict (cyber) bullying perpetration, and how do age, gender and nationality affect this relationship? Learning and Individual Differences, 45, 275-281. http:// dx.doi.org/10.1016/i.lindif.2015.11.021

Eisenberg, N. y Lennon, R. (1983). Sex differences in empathy and related capacities. Psychological Bulletin, 94, 100-131. http:// dx.doi.org/10.1037/0033-2909.94.1.100

Eisenberg, N., Fabes, R. A., Guthrie, I. K., y Reiser, M. (2000). Dispositional emotionality and regulation: their role in predicting quality of social functioning. Journal of Personality and Social Psychology, 78, 136-157. http:// dx.doi.org/10.1037/0022-3514.78.1.136

Euler, F., Steinlin, C., y Stadler, C. (2017). Distinct profiles of reactive and proactive aggression in adolescents: Associations with cognitive and affective empathy. Child and Adolescent Psychiatry and Mental Health, 17(1). http:// dx.doi.org/10.1186/s13034-016-0141-4

* Favre, D., Joly, J., Reynaud, C., y Salvador, L.L. (2009). Empathie, contagion émotionnelle et coupure par rapport aux émotions: validation d'un test pour repérer et aider des élèves à risque. Revue Européenne de Psychologie Appliquée, 59(3) 211-227. http://dx.doi. org/10.1016/i.erap.2009.03.004

* Forbes, E.L. (2003). Self-evaluation processes and the development of disruptive behavior problems: crosssectional and longitudinal perspectives in two cohorts of urban at-risk youths. Tesis doctoral. University of Rochester, New York. Recuperado de https://search.proquest.com/ docview/305309151? accountid $=17253$

* Freid, C. (2004). Restorative Justice and the development of empathy, remorse and moral disengagement in adolescent offenders. Tesis doctoral. Univeristy of Virginia, Virginia, EEUU.

García-López, L. M., del Campo, D., Gutiérrez, D., González-Víllora, S., y Valenzuela, A. V. (2012). Cambios en la empatía, la asertividad y las relaciones sociales por la aplicación del modelo de instrucción educación deportiva. Revista de Psicología del Deporte, 21(2), 321-330. Recuperado de https://search.proquest.com/ docview/1348793766? accountid = 17253

González-Gadea, M., Herrera, E., Parra, M., Méndez, P.G., Baez, S., Manes, F., y Ibáñez, A. (2014). Emotion recognition and cognitive empathy deficits in adolescent offenders revealed by context-sensitive tasks. Frontiers in Human Neuroscience, 8(11) 850. Recuperado de https://search.proquest.com/ docview/1685824010? accountid = 17253

* Goodman, G. (1998). The relationship among affect integration, emotion-engaged coping, empathy, and interpersonal violence in adolescents. Tesis doctoral. The Fielding Graduate Institute, Santa Barbara. Recuperado de https://search.proquest.com/ docview/304468596? accountid = 17253

Goutaudier, N., Lopez, S., Belarbi, A., Ducroux, M., y Chabrol, H. (2015). Traits de personnalité et comportements prosociaux chez les adolescents: Le rôle médiateur de I'empathie. Neuropsychiatrie De l'Enfance Et De l'Adolescence, 63(5), 310-316. http:// dx.doi.org/10.1016/i.neurenf.2014.11.007

* Granado, B. V. (2014). Emotional empathy: differences between adjudicated and nonadjudicated juvenile offenders. Tesis doctoral. Alliant International University, Los Angeles. Recuperado de https://search.proquest.com/ docview/1622146905? accountid = 17253

* Gray, L. (1997). Differences between violent and non-violent delinquent males on measures of empathy, attachment, parental criminality and parental acceptance/rejection. Tesis doctoral. University of Maryland, Maryland. Recuperado de https://search.proquest.com/ docview/304352531 ?accountid $=17253$

* Hackenberg, S.L. (2002). Empathy development and its relationship with aggressive and delinquent behavior in adolescents. Tesis doctoral. Cleveland State University, Ohio, EEUU. Recuperado de https://search.proquest.com/ docview/619951043? accountid = 17253

* Hepper, E.G., Hart, C.M., Meek, R., Cisek, 
S., y Sedikides, C. (2014). Narcissism and Empathy in Young Offenders and Nonoffenders. European Journal of Personality, 28(2), 201-210. http://dx.doi.org/10.1002/ per. 1939

Hoffman, M.L. (1977). Sex differences in empathy and related behaviors. Psychological Bulletin, 84, 712-722. http://dx.doi. org/10.1037/0033-2909.84.4.712

Hogan, R. (1969). Development of an Empathy Scale. Journal of Consulting and Clinical Psychology, 33, 307-316. http://dx.doi. org/10.1037/h0027580

* Horan, J.M. (2012). A prospective, longitudinal examination of the influence of childhood home and school contexts on psychopathic characteristics in adolescence. Tesis doctoral. Fordham University, New York. Recuperado de https://search.proquest.com/ docview/1518033052? accountid = 17253

*Hudak, M.A. (1978). DimensionsofDelinquency. Tesis doctoral. University of Wyoming, Laramie. Recuperado de https://search.proquest.com/ docview/302914488? accountid = 17253

* Hudak, M. A. (1980). Delinquency and social values: Differences between delinquent and nondelinquent adolescents. Youth \& Society, 17, 353-368. Recuperado de https://search.proquest.com/ docview/63726101? accountid $=17253$

* Hunter, J., Figueredo, A.J., Becker, J.V., y Malamuth, N. (2007). Non-sexual delinquency in juvenile sexual offenders: the mediating and moderating influences of emotional empathy. Journal of Family Violence, 22, 43-54. http:// dx.doi.org/10.1007/s10896-006-9056-9

* Hyde, L.W., Shaw, D.S., y Mollannen, K. L. (2010). Developmental precursors of moral disengagement and the role of moral disegagement in the development of antisocial behavior. Journal of Abnormal Child Psychology, 38(2), 197-209. http:// dx.doi.org/10.1007/s10802-009-9358-5

Jolliffe, D., y Farrington, D.P. (2004). Empathy and offending: a systematic review and metaanalysis. Aggression and Violent Behavior, 9(5), 441-476. http://dx.doi.org/10.1016/i. avb.2003.03.001
Jolliffe, D., y Farrington, D. P. (2006). Development and validation of the basic empathy scale. Journal of Adolescence, 29, 589-611. Recuperado de https://search.proquest.com/ docview/62109134? accountid $=17253$

* Jolliffe, D., y Farrington, D.P. (2007). Examining the relationship between low empathy and selfreported offending. Legal and Criminological Psychology, 12(2), 265-286. http://dx.doi. org/10.1348/135532506X147413

*Kaplan, P.yArbuthnot,J. (1985). Affectiveempathy and cognitive role-taking in delinquent and nondelinquent youth. Adolescence, 20, 323333. Recuperado de https://search.proquest. com/docview/62109134? accountid = 17253

* Kelley, D.M. (1992). Empathy and moral reasoning in adolescent sexual offenders. Tesis doctoral no publicada, United States International University, San Diego. Recuperado de https://search.proquest.com/ docview/304034923? accountid $=17253$

* Larden, M., Melin, L., Holst, U., y Langstrom, N. (2006). Moral judgement, cognitive distortions and empathy in incarcerated delinquent and community control adolescents. Psychology, Crime \& Law, 12(5), 453-462. http://dx.doi. $\mathrm{org} / 10.1080 / 10683160500036855$

* Lauterbach, O., y Hosser, D. (2007). Assessing empathy in prisoners- a shortened version of the Interpersonal Reactivity index. Swiss Journal of Psychology 66(2), 91-101. http:// dx.doi.org/10.1024/1421-0185.66.2.91

* Lee, M., y Prentice, N.M. (1988). Interrelations of empathy, cognition, and moral reasoning with dimensions of juvenile delinquency. Journal of Abnormal Child Pschology, 16(2), 127-139. http://dx.doi.org/10.1024/1421$\underline{0185.66 .2 .91}$

Li, X., Bian, C., Chen, Y., Huang, J., Ma, Y., Y Yu, Y. (2015). Development characteristics of empathy and its relationship with aggression in adolescents. Chinese Mental Health Journal, 29(9), 708-713. Recuperado de https://search.proquest.com/ docview/1734307180? accountid = 17253

* Lindsey, R. (1997). Dispositional empathy among juvenile sex offenders. Tesis doctoral. Oklahoma Sate University, Oklahoma, EEUU. 
Recuperado de https://search.proquest.com/ docview/304392570? accountid $=17253$

* Lindsey, R.E., Carlozzi, A.F., y Eells, G.T. (2001). Differences in the dispositional empathy of juvennile sex offenders, nonsex-offending delinquent juveniles, and nondelinquent juveniles. Journal of Interpersonal Violence, 16(6), 510-522. Recuperado de https://search.proquest.com/ docview/216838585? accountid $=17253$

* Lo, Q. H. Y. (2007). The relationship of selfcompassion and self-steem to empathy, shame, and social functioning in adolescents who offend sexually. Tesis doctoral. University of Toronto, Toronto, Canadá. Recuperado de https://search.proquest.com/ docview/304758412? accountid = 17253

* MacBride, G. H. (2005). Mental Health Recovery and psychopathy in a juvenile detention center. Tesis doctoral. University of Toledo, Ohio. Recuperado de https://search.proquest.com/ docview/621045827? accountid $=17253$

* Mak, A.S. (1991). Psychosocial control characteristics of delinquents and nondelinquents. Criminal Justice and Behavior, 18(3) 287-303. Recuperado de https://search.proquest.com/ docview/197248835? accountid = 17253

Martorell, C., González, R., Ordóñez, A., y Gómez, O. (2011). Estudio confirmatorio del cuestionario de conducta antisocial (CCA) y su relación con variables de personalidad y conducta antisocial. Revista Iberoamericana De Diagnóstico y Evaluación Psicológica, 31, 97-114. Recuperado de https://search.proquest.com/ docview/1151706187? accountid = 17253

* McCrady, F. (2005). Empathy and the adolescent sexualoffender: an examination of the specificity of empathy deficits and the relationship between empathy and distorted thought. Tesis doctoral. The Ohio State University. Ohio. Recuperado de https://search.proquest.com/ docview/621052779? accountid = 17253

* Milojevic, S., y Dimitrijevic, A. (2014). Empathic capacity of delinquent convicted minors. Psihologija, 47, 65-79. doi: http://dx.doi. org/10.2298/PSI1401065M
* Monto, M., Zgourides, G., Wilson, J., y Harris, R. (1994). Empathy and adolescent male sex-offenders. Perceptual and Motor Skills, 79(3), 1598. http://dx.doi.org/10.2466/ pms.1994.79.3f.1598

* Moriarty, N., Stough, C., Tidmarsh, P., Eger, D., y Dennison, S. (2001). Deficits in emotional intelligence underlying adolescent sexual offending. Journal of adolescence, 24, 743-751. Recuperado de https://search.proquest.com/ docview/21 1078691? accountid = 17253

Navas, E., Muñoz, J.J., y Graña, J.L. (2005). Influencia del género, edad y conducta antisocial en variables de personalidad. Revista de Psiquiatría de la Facultad de Medicina de Barcelona, 32, 57-64.

* Newton, N.C., y Bussey, K. (2012). The age of reason: An examination of psychosocial factors involved in delinquent behavior. Legal and Criminological Psychology, 17, 75-88. http://dx.doi.org/10.1111/i.20448333.2010.02004.x

* Noffsinger, M.A. (2006). Development of a multidimensional approach to understanding youthful offenders: The influence of psychosocial and personality risk factors. Tesis doctoral. University of North Texas, Texas, EEUU. Recuperado de https://search.proquest.com/ docview/305294795? accountid $=17253$

* Orozco-Truong, R. (1996). Empathy, guilt, and techniques of neutralization: Their role in a conceptual model of delinquent behavior. Tesis doctoral. University of Colorado, Colorado. Recuperado de https://search.proquest.com/ docview/304300090? accountid $=17253$

* Palucka, A. M. (1997). Relationships of moral judgement, emotional empathy and impulsivity to criminal behaviour in young and adult offenders. Tesis doctoral. Universidad de Toronto, Toronto, Canadá. Recuperado de https://search.proquest.com/ docview/619379667? accountid = 17253

* Park, J. (2003). Validating the effectiveness of a forgiveness intervention program for adolescent female aggressive victims in Korea. Tesis doctoral. University of Wisconsin-Madison, Wisconsin. Recuperado 
de https://search.proquest.com/ docview/305282929? accountid $=17253$

* Raymond, A. T. K., Overbeek, G., Minet, W., Rutger, C. M. E.E., y Scholte, R. H. J. (2007). Early adolescent empathy, parental support, and antisocial behavior. The Journal of Genetic Psychology, 168, 5-18. Recuperado de https://search.proquest.com/ docview/228458996? accountid $=17253$

* Reyes, L. (1990). A treatment program for juvenilehomicidial offenders: Impact on hostility-aggression, empathy and locus-of-control. Tesis doctoral. The University of Texas, Texas. Recuperado de https://search.proquest.com/ docview/303878792? accountid $=17253$

* Reynolds, J.R. (1999). A comparison of adolescentsexualoffenders, adolescent violent offenders, and adolescent non-violent, nonsexualoffendersalong the domains of empathy, hostility, and rape myth endorsement. Tesis doctoral. University of Missouri, Columbia. Recuperado de https://search.proquest.com/ docview/304511353? accountid = 17253

* Riley, J.A. (1986). Empathyand criminalbehavior: a look at man's inhumanity to man. Tesis doctoral. The Florida State University, Florida. Recuperado de https://search.proquest.com/ docview/303469538? accountid $=17253$

* Robinson, R., Roberts, W.L., Strayer, J., y Koopman, R. (2007). Empathy and emotional responsiveness in delinquent and non-delinquent adolescents. Social development, 16(3), 555-579. Recuperado de https://search.proquest.com/ docview/61953450? accountid $=17253$

Rosenthal, R. (1979). The file drawer problem and tolerance for null results. Psychological Bulletin, 86(3), 638-641. http://dx.doi. org/10.1037/0033-2909.86.3.638

* Saleem, Y.A., Brown, G., Withers, P., y Murphy, G. (2010). Investigation of empathy amongst offenders and non-offenders with ID. Journal of Applied Research in Intellectual disabilities, 23(5), 428-428.

Schwenck, C., Göhle, B., Hauf, J., Warnke, A., Freitag, C. M., y Schneider, W. (2014). Cognitive and emotional empathy in typically developing children: The influence of age, gender, and intelligence. European Journal of Developmental Psychology, 17(1), 63-76. http://dx.doi.org/10.1080/17405629.2013 .808994

* See, L., Ang, R.P., Loh, S., Fu, K.J., y Karre, J.K. (2011). The role of affective and empathy in psysical, verbal, and indirect aggression of a singaporean sample of boys. The Journal of Psychology: Interdisciplinary and Applied, 145(4), 313-330. http://dx.doi.org/10.1080 $\angle 00223980.2011 .568986$

Sobral, J. y Gómez-Fraguela, X.A. (2015). Notas para una Criminología de la voluntad. En F. Miró, J.R. Agustina, J.E. Medina, y L. Summers, (Eds.), Crimen, Oportunidad y Vida Diaria. Libro Homenaje al Profesor Dr. Marcus Felson (pp. 157-170). Madrid: Dykinson, S.L.

* Stams, G., Dekovic, M., Brugman, D., Rutten, E. A., Van den Wittenboer, G., Tavecchio, L.,... Van Schijndel, M. (2008). The relationship of punishment-and victim-based moral orientation to prosocial, externalizing, and norm trespassing behaviour in delinquent and non-delinquent adolescents: a validation study of the Moral Orientation Measure. Journal of Experimental Criminology, 4, 41-60. http:// dx.doi.org/10.1007/s 1 1292-007-9045-x

Stuiffzand, S., de Wied, M., Kempes, M., Van, d. G., Branje, S., y Meeus, W. (2016). Gender differences in empathic sadness towards persons of the same- versus other-sex during adolescence. Sex Roles, 75(9-10), 434-446. http://dx.doi.org/10.1007/s $11199-016-$ $\underline{0649-3}$

* Sturgeon, C.L. (2003). Typology of violence and alexithymia, empathy, perfectionism, and substance abuse in federal offenders. Tesis doctoral. York University, Toronto, Canadá. Recuperado de https://search.proquest.com/ docview/305288631? accountid $=17253$

* Thompson, R., Jones, D.J., Litrownik, A.J., English, D.J., Kotch, J.B., Lewis, T., y Dubowitz, H. (2014). Linking mother and youth parenting attitudes: Indirect effects via maltreatment, parent involvement, and youth functioning. Child Maltreatment, 19(3-4), 233-246. doi:http://dx.doi. 
org/10.1177/1077559514547263

* Trivits, L. (2005). A comparison of coping and coping resources in at-risk and seriously delinquent female adolescents. Tesis doctoral. University of Virginia, Virginia. Recuperado de from https://search.proquest.com/ docview/305414763? accountid $=17253$

Vachon, D.D., Lynam, D.R., y Johnson, J.A. (2014). The (Non) Relation between empathy and aggression: Surprising results from a meta-analysis. Psychological Bulletin, 140(3), 751-773. Recuperado de https://search.proquest.com/ docview/1523712848? accountid = 17253

* Van der Graaff, J., Branje, S., De Wled, M., y Meeus, W. (2012). The moderating role of empathy in the association between parental support and adolescent aggressive and delinquent behavior. Aggressive behavior, 38(5), 368-377. http://dx.doi.org/10.1002/ ab.21435

Van der Graaff, J., Branje, S., De Wied, M., Hawk, S., Van Lier, P., y Meeus, W. (2014). Perspective taking and empathic concern in adolescence: Gender differences in developmental changes. Developmental Psychology, 50(3), 881-888.

* Van Outsem, R., Beckett, R., Bullens, R., Vermeiren, R., Van Horn, J., y Doreleijers, T. (2006). The Adolescent Sexual Abuser Project (ASAP) assessment measures Dutch revised version: A comparison of personality characteristics between juvenile sex offenders, juvenile perpetrators of non-sexual violent offences and nondelinquent youth in the netherlands. Journal of Sexual Aggression, 12(2), 127-141. Recuperado de https://search.proquest.com/ docview/61899022? accountid $=17253$

* Way, I.F. (1999). Adolescent sexual offenders: the role of cognitive and emotional victim empathy in the victim-to-victimizer process. Tesis doctoral. Washington University, Missouri. Recuperado de https://search.proquest.com/ docview $/ 304533474$ ? accountid $=17253$ 